XX Міжнародний симпозіум «Методи дискретних особливостей в задачах математичної фізики/Discrete Singularities Methods in Mathematical Physics»,

УДК 62-137

МДОЗМФ/DSMMPh-2021

MSC 70E17

\title{
Calculation of the centrifulal pump critical frequencies based on its discrete mathematical model
}

\author{
A.Y. Verbovoy ${ }^{1}$, M.L. Sieryk ${ }^{2}$, I.V. Pavlenko ${ }^{3}$, A.A. Rudenko ${ }^{4}$ \\ ${ }^{1 ; 4}$ JSC «VNIIAEN», Sumy, Ukraine; ${ }^{2 ; 3}$ Sumy State University, Sumy, Ukraine \\ E-mail: \{ ${ }^{1}$ A.Verbovoy, ${ }^{3}$ I.Pavlennko\}@omdm.sumdu.edu.ua, \\ ${ }^{2}$ Maksus2010@icloud.com, ${ }^{4}$ Rudenko@vniiaen.sumy.ua
}

Calculations of the centrifugal machines critical frequencies of the rotors are currently carried out on the basis of the finite element method using multi-functional software as same as ANSYS and other similar, that work with finite elements of the threedimensional type, as well as some specialized computer programs working with the beam-type finite elements. Anyway, the finite element method is used. But in this case an user needs a lot of time for prepation of the initial data. Therefore, this article presents a calculation of the critical frequencies of the rotor of a centrifugal fuel pump of a liquidpropellant engine based on its discrete model. This calculation also includes an algorithm of results clarification. This model has been verificated by comparing the critical frequencies obtained in calculations based on the finite element beam model and discrete model with using the algorithm of results clarification and without it.

Key words: rotor dynamics, finite element model, centrifugal punp, discrete model of the rotor, vibrational state.

\section{Расчёт критических частот ротора центробежного насоса на ос- нове его дискретной математической модели}

\author{
А.Е. Вербовой ${ }^{1}$, М.Л. Серик ${ }^{2}$, И.В. Павленко ${ }^{3}$, А.А. Руденко ${ }^{4}$ \\ ${ }^{1 ; 4} \mathrm{AO}$ «ВНИИАЭН», Сумы, Украина; ${ }^{2 ; 3}$ Сумский государственный университет, Сумы, Ук- \\ раина \\ E-mail: $\left\{{ }^{1}\right.$ A.Verbovoy, ${ }^{3}$ I.Pavlennko\}@omdm.sumdu.edu.ua , \\ ${ }^{2}$ Maksus2010@icloud.com, ${ }^{4}$ Rudenko@vniiaen.sumy.ua
}

\begin{abstract}
Расчёты критических частот роторов центробежных машин в нынешнее время проводится на основе метода конечных элементов с использованием таких многоцелевых программных комплексов как, например, ANSYS и ему подобных, работающих с конечными элементами трёхмерного типа, а также некоторых специализированных компьютерных программ, работающих с конечными элементами балочного типа. Так или иначе, используется метод конечных элементов, для применения которого нужно большое количество времени на подготовку исходных данных. Поэтому в данной работе представлен расчёт критических частот ротора центробежного топливного насоса жидкостно-ракетного двигателя на основе его дискретной модели. Расчёт также включает в себя алгоритм уточнения полученных результатов. Также была проведена проверка достоверности модели путём сравнения критических частот, полученных при расчётах на основе конечно-элементной балочной модели и дискретной модели с и без применения алгоритма уточнения результатов.
\end{abstract}

Ключевые слова: динамика ротора, метод конечных элементов, центробежный наcoc, дискретная модель ротора, вибрационное состояние.

() Вербовой А.Е., Серик М.Л., Павленко И.В., Руденко А.А., 2021 


\section{1. Вступление}

Центробежные насосные агрегаты являются одним из наиболее употребляемых типов энергетического оборудования. Вопрос их вибрационной надёжности не перестаёт быть актуальным. Одним из условий обеспечения вибронадёжности центробежных насосов является отстройка от резонанса. При этом перед проведением соответствующих мероприятий необходимо, во-первых, определить критические частоты ротора. Для этого, как правило, используются специализированные компьютерные программы, основанные на реализации метода конечных элементов, использующие конечный элемент трёхмерного типа. Однако усложнение конструкции ротора и необходимость более точного определения критических частот в условиях учёта жёсткости подшипниковых опор и щелочных замедлений приводит к существенному увеличению подготовительного и машинного времени.

Таким образом, целью этой работы является устранение вышеупомянутых недостатков. Поставленная цель достигается путём разработки дискретной математической модели динамики ротора насоса горючего жидкостно-ракетного двигателя и соответствующего метода уточнения параметров расчёта. В результате оценены эквивалентные массы дискретной модели динамики ротора, а конечноэлементная модель сведена к эквивалентной трёхмассовой модели по критерию наилучшего совпадения собственных частот и форм свободных колебаний.

\section{2. Расчёт собственных частот и форм КЭ-модели}

Используем метод конечных элементов (МКЭ) для расчёта собственных частот и форм колебания ротора. Для этого воспользуемся программой «Critical frequencies of the rotor» созданной на основе МКЭ для балочных систем в системе компьютерной алгебры Mathcad $[1,2]$. Каждый участок ротора в программе описывается следующими параметрами: внутренним и внешним диаметром, длиной, коэффициентом жёсткости на краях участка и величине сосредоточенной точечной массы, плотностью материала, модулем упругости. Расчётная схема ротора с учётом всех вышеперечисленных параметров приведена на рисунке 1 .

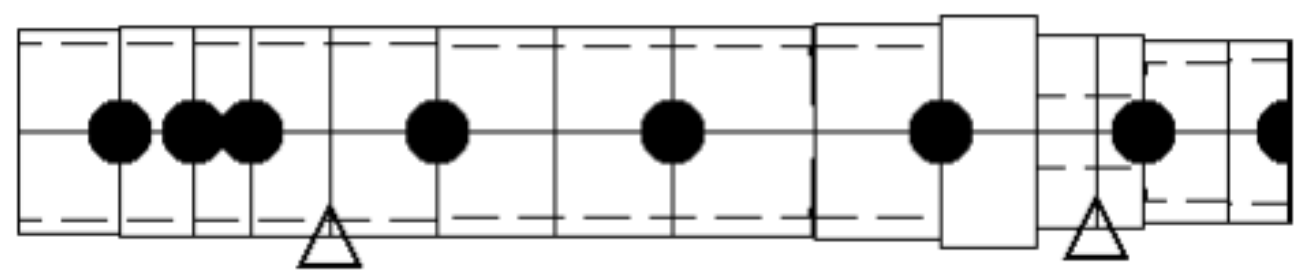

Рис. 1. Расчётная схема ротора

В результате получены первые три собственные частоты ротора.

$$
\omega=(3974 ; 5675 ; 7369) c^{-1}
$$

и соответствующие собственные формы. Графики собственных форм колебания ротора приведены на рисунке 2. 
XX Міжнародний симпозіум «Методи дискретних особливостей в задачах математичної фізики/Discrete Singularities Methods in Mathematical Physics», МДОЗМФ/DSMMPh-2021

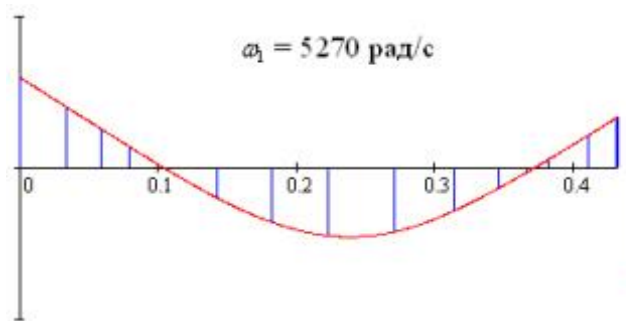

$a$

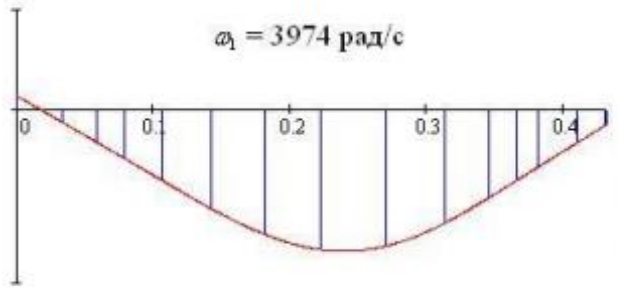

6

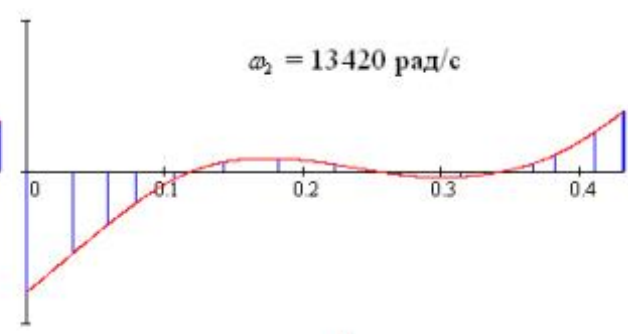

$\sigma$

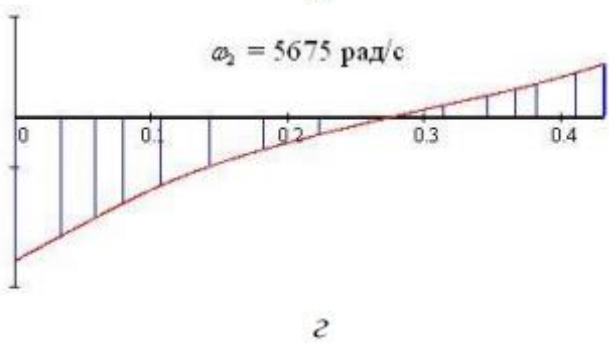

Рис. 2. Собственные формы колебаний

Формы «а» и «в» на рисунке 2 - первые собственная форма колебаний; «б» и «г» - вторые.

Формы «а» и «б» на рисунке 2 - формы колебаний для модели типа «bonded», a «в» и «г» - для модели типа «frictional» в плане контактного взаимодействия тел.

\section{3. Расчёт эквивалентных масс 3-массовой дискретной модели ротора}

Схема 3-массовой дискретной модели ротора представлена на рисунке 3. Используя конечно-элементную модель (КЭ-модель) были получены коэффициенты влияния сил приложенных в ј-й точке на перемещение i-й точки. Коэффициент определяется с помощью программы расчёта вынужденных колебаний ротора, как прогиб ротора в і-й точке при приложенном в ј-й точке единичного дисбаланса и при частоте вращения ротора.

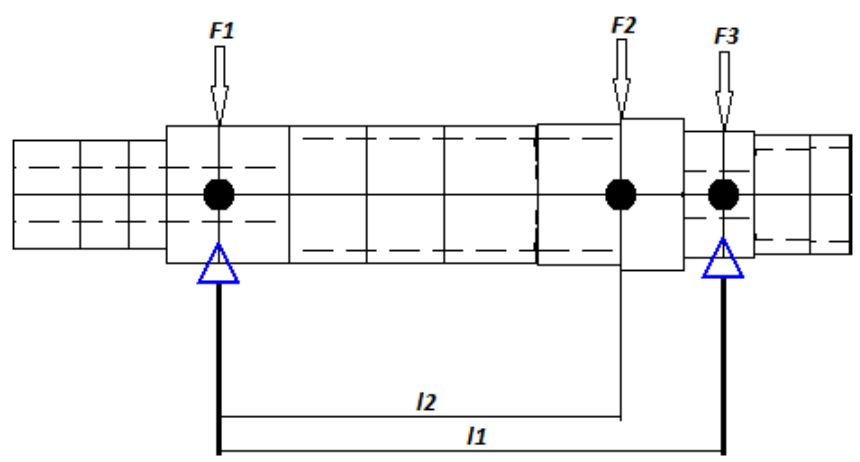

Рис. 3. Схема трёхмассовой дискретной модели ротора 
Запишем систему дифференциальных уравнений свободных колебаний ротора в обратной форме:

$$
\left.\begin{array}{l}
x_{1}=\beta_{11} F_{1}+\beta_{12} F_{2}+\beta_{13} F_{3} \\
x_{2}=\beta_{21} F_{1}+\beta_{22} F_{2}+\beta_{23} F_{3} \\
x_{3}=\beta_{31} F_{1}+\beta_{32} F_{2}+\beta_{33} F_{3}
\end{array}\right\} .
$$

В последние соотношения, используя принцип Д'Аламбера, подставим следующее равенство:

$$
F_{j}=-m_{j} \cdot \ddot{x}_{j} .
$$

В результате подстановки имеем дифференциальные уравнения (3) свободных колебаний линейной трёхмассовой модели ротора.

$$
\left.\begin{array}{l}
x_{1}=-\beta_{11} m_{1} \cdot \ddot{x}_{1}-\beta_{12} m_{2} \cdot \ddot{x}_{2}-\beta_{13} m_{3} \cdot \ddot{x}_{3} \\
x_{2}=-\beta_{21} m_{1} \cdot \ddot{x}_{1}-\beta_{22} m_{2} \cdot \ddot{x}_{2}-\beta_{23} m_{3} \cdot \ddot{x}_{3} \\
x_{3}=-\beta_{31} m_{1} \cdot \ddot{x}_{1}-\beta_{32} m_{2} \cdot \ddot{x}_{2}-\beta_{33} m_{3} \cdot \ddot{x}_{3}
\end{array}\right\} .
$$

Для нахождения решения колебаний дискретных масс представим в виде:

$$
x_{i}=B \sin (\omega t) \text {. }
$$

Подставив (4) в (3) и сократив на, имеем

$$
\left.\begin{array}{l}
B_{1}=-\beta_{11} m_{1} \cdot B_{1} \omega^{2}-\beta_{12} m_{2} \cdot B_{2} \omega^{2}-\beta_{13} m_{3} \cdot B_{3} \omega^{2} \\
B_{2}=-\beta_{21} m_{1} \cdot B_{1} \omega^{2}-\beta_{22} m_{2} \cdot B_{2} \omega^{2}-\beta_{23} m_{3} \cdot B_{3} \omega^{2} \\
B_{3}=-\beta_{31} m_{1} \cdot B_{1} \omega_{1}^{2}-\beta_{32} m_{2} \cdot B_{2} \omega^{2}-\beta_{33} m_{3} \cdot B_{3} \omega^{2}
\end{array}\right\} .
$$

Введём замену $\omega^{2}=z$ и сгруппируем:

$$
\left.\begin{array}{l}
\left(\beta_{11} m_{1}-z\right) B_{1}+\beta_{12} m_{2} B_{2}+\beta_{13} m_{3} B_{3}=0 \\
\beta_{21} m_{1} B_{1}+\left(\beta_{22} m_{2}-z\right) B_{2}+\beta_{23} m_{3} B_{3}=0 \\
\beta_{31} m_{1} B_{1}+\beta_{23} m_{2} B_{2}+\left(\beta_{33} m_{3}-z\right) B_{3}=0
\end{array}\right\} .
$$

Сократив систему уравнений (6) на $\mathrm{B}$, придём к виду:

$$
\left.\begin{array}{l}
\left(\beta_{11} m_{1}-z\right)+\beta_{12} m_{2} U_{2}+\beta_{13} m_{3} U_{3}=0 \\
\beta_{21} m_{1} U_{1}+\left(\beta_{22} m_{2}-z\right)+\beta_{23} m_{3} U_{3}=0 \\
\beta_{31} m_{1} U_{1}+\beta_{23} m_{2} U_{2}+\left(\beta_{33} m_{3}-z\right)=0
\end{array}\right\} .
$$

Поскольку из расчёта КЭ-модели теперь известны собственные частоты и относительные амплитуды свободных колебаний на собственных частотах (номер массы; - номер собственной частоты), можно рассматривать задачу оценивания, за параметры которой выберем массы дискретной модели (4).

Перепишем (7) в следующем виде:

$$
\bar{D} \cdot \bar{m}=\bar{C}
$$

где $\bar{D}$ - матрица размерностью $(9 \times 3)$ : 
XX Міжнародний симпозіум «Методи дискретних особливостей в задачах математичної фізики/Discrete Singularities Methods in Mathematical Physics»,

МДОЗМФ/DSMМPh-2021

$$
\bar{D}=\left[\begin{array}{lll}
\beta_{11} U_{1}^{(1)} & \beta_{12} U_{1}^{(1)} & \beta_{13} U_{1}^{(1)} \\
\beta_{11} U_{2}^{(1)} & \beta_{22} U_{2}^{(1)} & \beta_{23} U_{2}^{(1)} \\
\beta_{11} U_{3}^{(1)} & \beta_{32} U_{3}^{(1)} & \beta_{33} U_{3}^{(1)} \\
\beta_{11} U_{1}^{(2)} & \beta_{12} U_{1}^{(2)} & \beta_{13} U_{1}^{(2)} \\
\beta_{11} U_{2}^{(2)} & \beta_{22} U_{2}^{(2)} & \beta_{23} U_{2}^{(2)} \\
\beta_{11} U_{3}^{(2)} & \beta_{32} U_{3}^{(2)} & \beta_{33} U_{3}^{(2)} \\
\beta_{11} U_{1}^{(3)} & \beta_{12} U_{1}^{(3)} & \beta_{13} U_{1}^{(3)} \\
\beta_{11} U_{2}^{(3)} & \beta_{22} U_{2}^{(3)} & \beta_{23} U_{2}^{(3)} \\
\beta_{11} U_{3}^{(3)} & \beta_{32} U_{3}^{(3)} & \beta_{33} U_{3}^{(3)}
\end{array}\right],
$$

$\bar{m}$ - вектор-столбец эквивалентных масс размерностью (9х3):

$$
\bar{m}=\left[\begin{array}{l}
m_{1} \\
m_{2} \\
m_{3}
\end{array}\right],
$$

$\bar{C}$ - вектор-столбец правых частей размерностью (9x1):

$$
\bar{C}=\left[\begin{array}{c}
z_{1} U_{1}^{(1)} \\
z_{1} U_{2}^{(1)} \\
z_{1} U_{3}^{(1)} \\
z_{1} U_{1}^{(2)} \\
z_{1} U_{2}^{(2)} \\
z_{1} U_{3}^{(2)} \\
z_{1} U_{1}^{(3)} \\
z_{1} U_{2}^{(3)} \\
z_{1} U_{3}^{(3)}
\end{array}\right] .
$$

С помощью формулы линейной регрессии

$$
\bar{m}=\left[\bar{D}^{T} \bar{D}\right]^{-1} \bar{D}^{T} \bar{C}
$$

были получены следующие значения величин эквивалентных масс ротора:

$$
\bar{m}=\left(\begin{array}{lll}
8,055 & 6,375 \quad 0,765
\end{array}\right)
$$

Адекватность построенной 3-массовой модели ротора была проверена с помощью сравнений значений критических (собственных) колебаний ротора. Погрешности в значениях частот СЕ-модели и 3 -массовой определялись по формуле (14):

$$
\varepsilon_{k}=\frac{\omega_{k}-\omega_{k}^{*}}{\omega_{k}^{*}},
$$


где $\omega_{k}^{*}$ - соответствующая критическая частота колебаний ротора КЭ-модели, $\omega_{k}$ - критическая частота колебаний дискретной 3-массовой модели.

Из условия нетривиального решения системы (7) относительно амплитуд , найдем критические (собственные) частоты колебаний дискретной трехмассовой модели из уравнения (7):

$$
\Delta(z)=\left[\begin{array}{ccc}
\beta_{11} m_{1}-z & \beta_{12} m_{2} & \beta_{13} m_{3} \\
\beta_{21} m_{1} & \beta_{22} m_{2}-z & \beta_{23} m_{3} \\
\beta_{31} m_{1} & \beta_{23} m_{2} & \beta_{33} m_{3}-z
\end{array}\right]=0 .
$$

В результате решения системы (15) были найдены собственные частоты колебаний дискретной трёхмассовой модели:

$$
\omega_{k}=\left(\begin{array}{lll}
4406 & 5407 & 12610
\end{array}\right) c^{-1} .
$$

Соответствующие критические частоты колебаний ротора СЕ-модели:

$$
\omega_{k}^{*}=\left(\begin{array}{lll}
3878 & 5597 & 7821
\end{array}\right) c^{-1} .
$$

Подставив полученные значения в уравнение (4.13) имеем следующие значения величины погрешности:

$$
\varepsilon=(13,6 \quad 3,40 \quad 61,2) \%
$$

Были оценены погрешности расчёта критических (собственных) частот КЭмодели и 3-массовой дискретной модели (таблица 1).

Таблица 1. Сравнение результатов расчёта

\begin{tabular}{|c|c|c|c|}
\hline \multirow{2}{*}{ Порядковый номер } & \multicolumn{2}{|c|}{ Критические частоты, рад/с } & \multirow{2}{*}{ Разность, \% } \\
\cline { 2 - 3 } & $\begin{array}{c}\text { Программа } \\
\text { «Сritical» }\end{array}$ & 3-х массовая & \\
\hline 1 & 3878 & 4406 & 13,6 \\
\hline 2 & 5597 & 5407 & 3,40 \\
\hline 3 & 7821 & 12610 & 61,2 \\
\hline
\end{tabular}

\section{4. Метод уточнения результатов расчёта на основе дискретной модели}

Поскольку погрешность возрастает с ростом собственной частоты, то существует необходимость уточнения результатов расчёта. Возьмём матрицу коэффициентов податливости, и проведём серию виртуальных экспериментов, количество которых было задано значением $\mathrm{N}=10000$.

А теперь рассмотрим алгоритм, который был применён для расчёта. Для начала нужно было взять нулевой вектор масс и нулевой вектор собственных частот. Оценив полученные массы, взятые из (13) предыдущего пункта, запишем в следующие выражения:

$$
\begin{aligned}
& \mathrm{m}_{1} \leftarrow 6+\mathrm{md}(4) \\
& \mathrm{m}_{2} \leftarrow 4+\operatorname{md}(4) \\
& \mathrm{m}_{3} \leftarrow 0.6+\mathrm{md}(0.4)
\end{aligned}
$$


XX Міжнародний симпозіум «Методи дискретних особливостей в задачах математичної фізики/Discrete Singularities Methods in Mathematical Physics»,

\section{МДОЗМФ/DSMМPh-2021}

Следующим шагом было проведено объединение нулевой матрицы М и коэффициентов $\mathrm{m}_{1}, \mathrm{~m}_{2}, \mathrm{~m}_{3}$. Из дискретной 3-х массовой модели, для неё создано частотное уравнение

$$
\left|\begin{array}{ccc}
\delta_{11} m_{1}-z & \delta_{12} m_{2} & \delta_{13} m_{3} \\
\delta_{21} m_{1} & \delta_{22} m_{2}-z & \delta_{23} m_{3} \\
\delta_{31} m_{1} & \delta_{23} m_{2} & \delta_{33} m_{3}-z
\end{array}\right|=0
$$

и получили кубический полином от z.

С помощью оператора polyroots были найдены корни полинома.

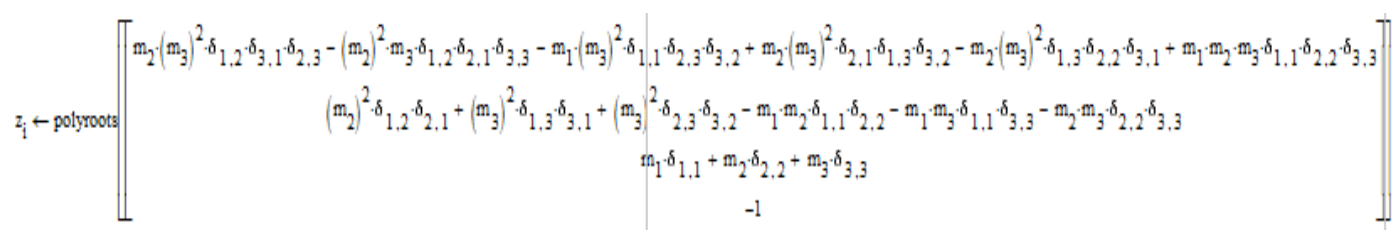

Рис. 4. Корни уравнения (19) относительно z (снимок экрана)

А по формуле (20) нашли значение и объединили полученные значения в матрицу с предварительно заданными нулевыми.

$$
\omega=\frac{1}{\sqrt{z}}
$$

Данный алгоритм будет повторяться 10000 раз.

Далее матрицы масс и собственных частот объединились в одну и выделились полученные результаты в отдельную матрицу начиная с 2 строки (1 строканулевые значения) до и соответственно Столбцы от 1 до 6.

Для нахождения уточнённых масс из полученной матрицы, находим ту строку, в которой найденные собственные частоты будут максимально близкими к данным:

$$
\omega_{k}^{*}=\left(\begin{array}{lll}
3878 & 5597 & 7821
\end{array}\right) c^{-1},
$$

чтобы выбрать такой из полученных результатов записали такую формулу:

$$
R_{i}=\sum_{j=1}^{3}\left(\alpha_{j}\left(\frac{\omega_{i j}}{\omega_{0 j}}-1\right)^{2}\right)
$$

где $\alpha_{j}$ - коэффициент влиятельности.

Вместо численного вывода результата, был использован графический метод. Больше всего подходят наименьшие полученные значения, которые мы нашли с помощью трассировки. Подставим значение $\mathrm{k}=6099$ и получим уточнённые массы та собственные частоты:

$$
\begin{aligned}
\bar{m}_{y m} & =\left(\begin{array}{lll}
7,701 & 7,960 & 0,994
\end{array}\right) \kappa 2 \\
\bar{\omega}_{y m} & =\left(\begin{array}{lll}
3970 & 5503 & 11100
\end{array}\right) c^{-1}
\end{aligned}
$$




\section{5. Сравнение результатов}

По сравнению с предварительно полученными результатами, а именно это сравнение представлено в виде таблицы 2.

Таблица 2. Сравнение результатов расчёта

\begin{tabular}{|c|c|c|c|c|c|}
\hline \multirow{2}{*}{$\begin{array}{c}\text { Порядковый } \\
\text { номер }\end{array}$} & $\begin{array}{c}\text { Программа } \\
\text { «Critical» }\end{array}$ & $\begin{array}{c}\text { К-х массовая } \\
\text { модель }\end{array}$ & $\begin{array}{c}\text { 3-х массовая } \\
\text { модель с } \\
\text { уточнением }\end{array}$ & $\begin{array}{c}\text { Программа } \\
\text { «Critical» }\end{array}$ & $\begin{array}{c}\text { 3-х массовая } \\
\text { модель }\end{array}$ \\
\hline 1 & 3878 & 4406 & 1 & 3878 & 4406 \\
\hline 2 & 5597 & 5407 & 2 & 5597 & 5407 \\
\hline 3 & 7821 & 12610 & 3 & 7821 & 12610 \\
\hline
\end{tabular}

Здесь можно увидеть, насколько улучшилась ситуация с погрешностью в расчёте благодаря данному методу. Погрешности расчёта критических (собственных) частот КЭ-модели и 3-массовой дискретной модели. Для первых трех критических частот величина погрешности составляла $13.62 \%, 3.4 \%$ и $61.25 \%$.

После расчёта по методу уточнения погрешность составляет $2.38 \%, 1.68 \%$ и $41.96 \%$. А это почти в 6 раз меньше для первой собственной частоты, в 2 раза меньше для второй и в 1,5 раза меньше для третей.

\section{6. Выводы}

В результате проведённой работы были получены собственные частоты и формы для насоса горючего в двух вариациях: «frictional» и «bonded». В первом варианте значения жёсткости подшипников принимались минимальными. Во втором варианте брались максимально возможные жёсткости.

Расчёт критических частот колебаний многомассовой модели ротора осуществлялся с помощью рабочего файла «Critical frequencies of the rotor». Как результат проведения уточняющих расчётов для дискретной модели, определена степень её достоверности путём сравнения с соответствующими результатами конечно-элементной модели. В частности, относительная погрешность определения первых двух критических частот не превышает 2,5\%. Для уменьшения погрешности расчёта третьей критической частоты предлагается применять как взвешенный метод наименьших квадратов, так и системы расчётного интеллекта в гармоничном сочетании с методами теории принятия решений.

Таким образом, создана достоверная трёхмассовая математическая модель динамики ротора, основанная на комплексном применении метода конечных элементов и теории колебаний, а также разработан алгоритм уточнения результатов расчёта. Предложенная методика может быть использована для отстройки от резонанса гибких роторов турбонасосных агрегатов жидкостных ракетных двигателей.

\section{ЛИТЕРАТУРА}

1. Комп'ютерна програма "Critical frequencies of the rotor": авторське свідоцтво №59855, Україна. I.В. Павленко, В.І. Симоновський. Дата реєстрації 27.05.2015.

2. Програмне забезпечення Mathcad Prime 3.1 Profes, ліцензійна угода № 216/16/1000, дата придбання 24.11.2016. 
XX Міжнародний симпозіум «Методи дискретних особливостей в задачах математичної фізики/Discrete Singularities Methods in Mathematical Physics», МДОЗМФ/DSMМPh-2021

3. Verbovyi A., Neamtu C., Sieryk M. et al. Ensuring the Vibration Reliability of Rotors Connected by Spline Joints. Журнал інженерних наук. 2019. T. 6, № 2. C. D14-D19. doi:10.21272/jes.2019.6(2).d3

4. Pavlenko I.V., Simonovskiy V., Pitel' J., Demianenko M.M., Verbovyi A.Ye. Investigation of non-linear reactions in rotors' bearing supports of turbo-pump units for liquid rocket engines. Journal of Engineering Sciences. 2018. Volume 5, Issue 1. P. D6-D14. doi:10.21272/jes.2018.5(1).d2

5. Pavlenko I., Demyanenko M., Simonovskiy V., Pitel' J., Pavlenko V., Verbovyi A. Comprehensive approach for identification of nonlinear stiffness characteristics of bearing supports for the oxidizer turbopump of the liquid rocket engine. Journal of Engineering Sciences. 2018. Volume 5, Issue 2. P. D6-D14. doi:10.21272/jes.2018.5(2).d2

6. Yashchenko A., Verbovyi A., Rudenko A. Computational study of the effect of pump mounting to foundation compliance on pump natural frequencies. Journal of Physics: Conference Series. 2021, vol. 1741, p. 012042. doi: 10.1088/17426596/1741/1/012042

7. Yashchenko A., Verbovyi A., Rudenko A. Effect of pump duty on natural frequencies and modes of its rotor vibration. Journal of Physics: Conference Series. 2021, vol. 1741, p. 012041. doi:10.1088/1742-6596/1741/1/012041

8. Pavlenko I.V., Simonovsky V.I., Pitel' J., Verbovyi A.E., Demianenko M.M. Investigation of critical frequencies of the centrifugal compressor rotor with taking into account stiffness of bearings and seals. Journal of Engineering Sciences. 2017. Volume 4, Issue 1. P. C1-C6.

9. Pavlenko I.V., Siminovskiy V.I., Demianenko M.M. Dynamic analysis of centrifugal machines rotors supported on ball bearings by combined application of $3 \mathrm{D}$ and beam finite element models. IOP Cenference Series: Materials Science and Engineering, 2017. Vol. 233(1), 012053, doi:10.1088/1757-899X/233/1/012053

10. Санин А.И., Симоновский В.И. Анализ нелинейных колебаний ротора центробежного насоса. Сучасні технології у промисловому виробництві : матеріали науково-технічної конференції викладачів, співробітників, аспірантів і студентів фак-ту техн. систем та енергоефективних технологій, м. Суми, 18-21 квітня 2017 р.: у 2-х ч. Суми: СумДУ, 2017. Ч.1. С. 154.

Надійшла 30.05.2021. 


\title{
Розрахунок критичних частот ротора відцентрового насоса на основі його дискретної математичної моделі
}

\author{
А.С. Вербовий ${ }^{1}$, М.Л. Сєрик ${ }^{2}$ І.В. Павленко ${ }^{3}$, А.А. Руденко ${ }^{4}$ \\ ${ }^{1 ; 4} \mathrm{AT}$ «ВНИИАЭН», Суми, Україна; ${ }^{2 ; 3}$ Сумський державний університет, Сумы, Украина \\ E-mail: \{ ${ }^{1}$ A.Verbovoy, ${ }^{3}$ I.Pavlennko\}@omdm.sumdu.edu.ua, \\ ${ }^{2}$ Maksus2010@icloud.com, ${ }^{4}$ Rudenko@vniiaen.sumy.ua

\begin{abstract}
Розрахунки критичних частот роторів відцентрових машин в нинішній час проводиться на основі методу кінцевих елементів 3 використанням таких багатоцільових програмних комплексів як, наприклад, ANSYS і йому подібних, що працюють 3 кінцевими елементами тривимірного типу, а також деяких спеціалізованих комп'ютерних програм, що працюють 3 кінцевими елементами балочного типу. Так чи інакше, використовується метод кінцевих елементів, для застосування якого потрібна велика кількість часу на підготовку вихідних даних. Тому в даній роботі представлений розрахунок критичних частот ротора відцентрового паливного насоса рідинно-ракетного двигуна на основі його дискретної моделі. Розрахунок також включає в себе алгоритм уточнення отриманих результатів. Також була проведена перевірка достовірності моделі шляхом порівняння критичних частот, отриманих при розрахунках на основі конечно-елементної балкової моделі і дискретної моделі з та без застосування алгоритму уточнення результатів.
\end{abstract}

Ключові слова: динаміка ротора, метод скінченних элементів, віцентровий насос, дискретна модель ротора, вібраціний стан. 